\title{
Enfermedad de Cushing en un niño de 12 meses
}

\author{
Dr Fonald Youlton
}

\begin{abstract}
A 12 month old by with Cushing's disedse is reported. He had the classical features of hipercortisolism, elevevated plasma corticosol levels that did not suppress with dexamethasone, increased excretion of urinary $1 \vec{f}$ OHCS and an enlarged sella turcica. He died shortly after admission. Necropsy findings included as istracranial hemorrage, a pituitary tunour, with the characteristics of a chrumophobe adenoma, and bilateral adrenal lyperplasia.
\end{abstract}

El sindrome de Cushing es una afección poco común durante la infancia. ${ }^{\prime}$ En el niño mayor, al igual yue en el adulto, predominan atquellos casos debido a hjpersecreción de ACTH. ${ }^{2}$ En el lactante, sin embargo, más del $80 \%$ de los casos son originados por adenomas o carcinomas suprarrenales y menos del $10 \%$ lo son por hiperplasia suprarrenal difusa, secundaria a alteraciones hipotálamo-hipofisiarias. ${ }^{3.4,5}$

Se presenta un caso de esta última variedad con comprobación anatomopatológica.

\section{CASO CLINICO}

CBM de 12 meses de edad, fue pronducto de un embarazo normal y parto cesárea. Pesú $4130 \mathrm{gr}$. y midió $55 \mathrm{~cm}$. al nacer.

Su crecimiento y desarrollo fueron aparentemente normales durante el primer semestre de vida. Desde los 8 meses de edad, sus padres notaron aumento del apetitu y del peso, hiperpigmentación de la piel, hipertricorsis facial y dorsal, auné de la frente y mentón y moderado crecimiento del pene y escroto. A estos signos, que fueron progresivos, se agregaron irritabilidad e insomnio. Tres días antes de su ingreso presentó cuadro respiratorio febril, durante el que se aitemaron periodos de excitación y sumnolencia, luego mirada vaga y rigidez de] cuello, siendo hosptalizado a los 12 meses de edad.

El examen fisico mostró un lactante en estado grave, febril, irritable, paco conectado con el medio ambiente, con obesidad yeneralizada, pero muy marcada a nivel de las mejillas y dorso; facies pletórica. Había intensa

Servicio de Cenética, Hospital J.J. Aguirre y Unidad de Endor crinologia, Hospital Roberto del Hio, Santiago. pigmentación de la piel, la que estaba sudorosa. La hipertricosis generalizada era mayor en frente y mejillas, donde destaciaban elementos de comedones $y$ acrié $\{\mathbf{F}$ gura 1). Había marcados signos de irritación meníngea. Su tulla era $73 \mathrm{~cm} .(-1.0 \mathrm{DS})$, su peso $12.6 \mathrm{Kg}(+20 \mathrm{DS})$ y el perímetro craneano, $49.8 \mathrm{~cm}$. $(+2.2 \mathrm{DS})$. La presión arterial era $160 / 80 \mathrm{~mm} \mathrm{Hy}$.

Las punciones lumbares efectuladas al ingreso y al día siguiente dieron salida a LCR hemontágico; los exámenes bacteriológicos de estas muestras al igual que los hemocultivos fueron negativos.

El hematocrito era $30.5 \%$, el recuento de leucocitos $17.500 \times \mathrm{mm}^{3} \sin$ desviación a izquierda y la VHS, 100 $\mathrm{mm}$ en la primera hora. El sedimento urinario, la glicemia y los electrólitos plasmáticos estaban dentro de los valores normales.

Durante su hospitalización el paciente presentó polidipsia y poliuria de hasta $130 \mathrm{ml}$. de orina $\mathbf{x} \mathbf{K g}$. de peso. La osmularidad plasmática en dos oxasiones fue 315 m0sm.L y las de la orina obtenidas en forma simultánea fueron 483 y $604 \mathrm{~m} / \mathrm{l}$.

La radiografía de cráneu mostró una silla turca grande y excavada.

Las deteminaciones hormonales realizadas en el paciente se muestran en las tablas 1 y $2 *$

La hipertensión arterial se mantuvo. Al noveno día de hospital ización, el paciente presentó convulsiones tónico-clónicas generalizadas y aparente amaurosis, falleciendo al 11 dia El diagnóstico clínico fue Síndrome de Clsshing, tumor hipofisiario, hemonagía subaracnoidea.

La necropsia (nealizada por los Dres. Ricardo Rojas M. y Sergio Scroggie A.) mostró, en lo fundamental, lo siguiente: encéfalo edematoso; meninges hiperhèmicas con pequeños focos hemorrágicos en ambos hernisfe-

*Todas las determinaciones hormonales por radioinnunounálisis lueron efectuadas gentilmente en el Laboratorio de la Dra. Teresa Palma. 


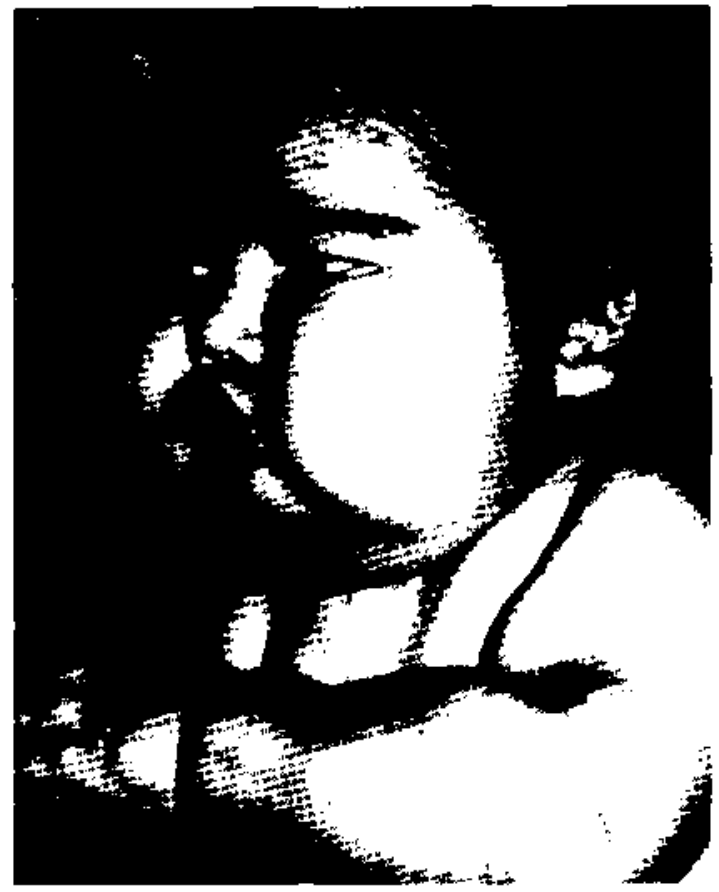

Figura 1.

rios; dilatación de ambos ventriculos laterales, el derecho con hemorraglia rue ocupa la mitad del ventriculo; sill a turca de $2.5 \times 2.5$ ertr. con destrucción de las apófisis clinoides; hipófísis aumentada de tamaño y consistencia, de aspectu hemomágico, de $4.0 \times 2.5 \times 2.5 \mathrm{~cm}$, com-

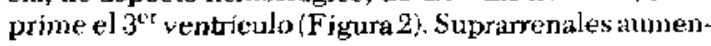
tadas a expensas de la corteza, la derecha de $5.5 \mathrm{gr}$ y la isquierda de $3.5 \mathrm{gr}$.
Tabla N." i

C.B.M. R. del R. N." 547299

Síndronı de Cuslıing

- Cortisol plastrkitico

$\begin{array}{ll}\text { AMI } & 46 \\ \text { PM } & 48 \\ \text { AM post 1)ex. } & 38\end{array}$

$\mu \mathrm{g} / \mathrm{d} !$ $\mu \mathrm{g} / \mathrm{d} \mathrm{j}$ $\mu \mathrm{g} f \mathrm{dl}$

- 17 OHCS urinarios $=11.3 \mathrm{mg} / 24 \mathrm{hrs}$.

$-17 \mathrm{Ks}$. urinarios $=2.32 \mathrm{mg} / 24 \mathrm{hrs}$.

Tabla N. ${ }^{\circ}$

C.B.M.

R. del R. N."547299

Sindrome de Cushing

Yiveles Hormonites

〈plasria)

- HGH

1.5

- PROL.

$-\mathrm{LH}$

- FSH

3.6

$-\mathrm{T}_{4}$

4.8

- TSH

4.2

- TSH $\mathrm{ng} / \mathrm{ml}$ $\mathrm{ng} / \mathrm{ml}$ $\mathrm{mIU} / \mathrm{ml}$ $\mathrm{mIU} / \mathrm{ml}$ ugtd $\mu \mathrm{L} / \mathrm{dl}$ $\mu \mathrm{U} / \mathbf{m l}$

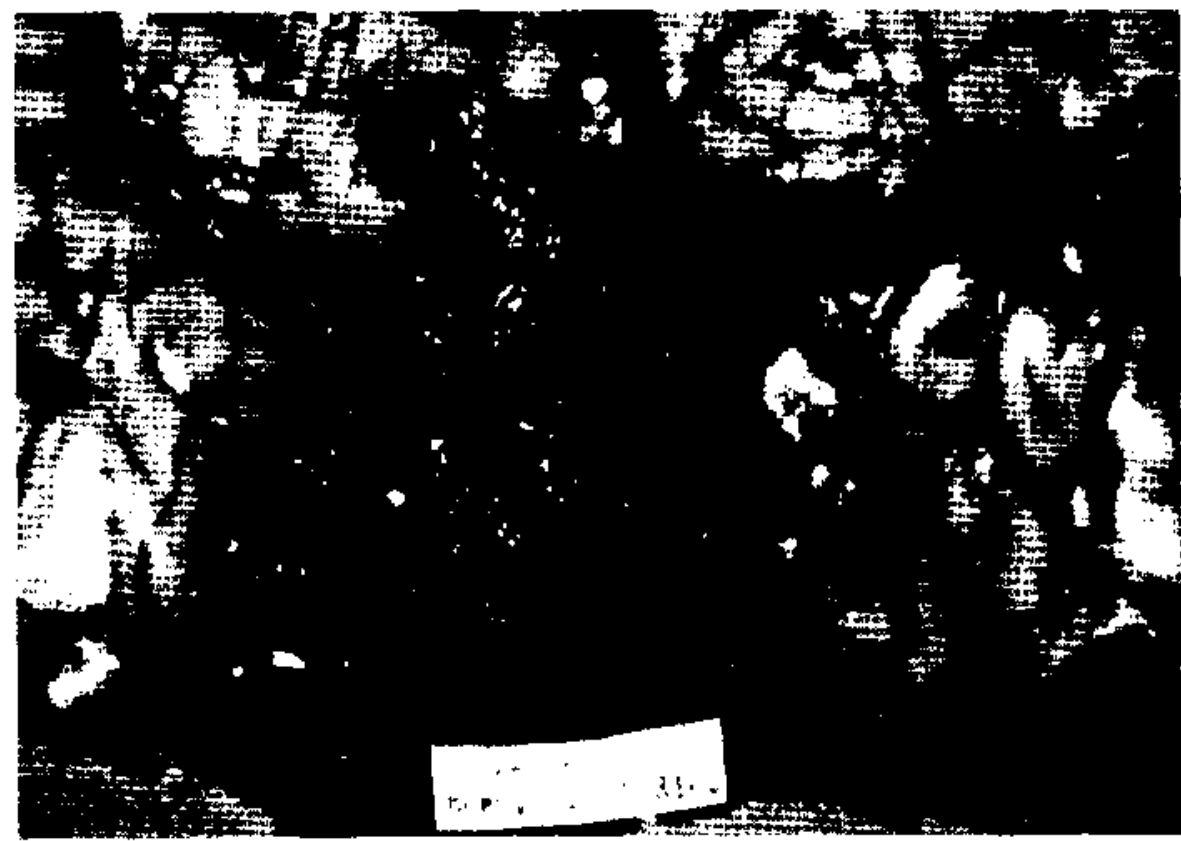

Figura 2. 
El estudio histológico demostró hiperplasia de las suprarrenales y ura hipófisis con intensa proliferación neoplasica de células pequeñas sin o con escasa grasulación, con caracteres de adenoma cromófobo.

\section{COMENTARIO}

Los síntomas y signos presentes en nuestro enfermo son característicos del $\mathbf{S}$. de Cushing. Por los antecedentes, éste se habria instalado en el segundo semestre de vida. La talla de nacimiento estaba 2.5 D.S. sobre el promedio y al momento de consultaroos, 1.0 D.S. por debajo. No contamos con mediciones previas que habrian sido útiles para establecer la edad en que se produjo la desaceleración del crecimiento, signo destacado del hipercortisolismo en la infancia..$^{6.7}$

La obesidad a esta edad es más generalizada que en el adulto y el abultamiento de las mejillas es más exagerado. Si bien la obesidad es un problema frecuente en lactantes normales, ésta se acompaña de un ritmo de crecimiento normal o acelerado. La medición de la presión arterial en estos casos es un elemento clínico orientador.

La precoz aparición de hipertricosis y acné faciales, deben hacer pensar en la existencia de una fuente anomal de andrógenos, al igual que el crecimiento del pene y escroto. La palpación de testículos de tamaño nomsal apunta hacia una causa suprarrenàl.

La hiperpignentación de la piel puede ser ubservable sólo en los $\mathbf{\$}$. de Cushing hipofisiarios o ectópicos y se debe tanto a la hipersecreción de ACTH como posiblemente también de Lipotropina (LPH), molécula precursora de $\beta$ MSH y que a su vez se origina de un precursor común con ACTH. ${ }^{2}$

Lai irritabilidad e insomnio manifestados por nuestro paciente pueden ser en parte explicados por el hipercurtisolismo, pero más probablemente fieron debidos a hipertensión intracraneana. Esta tiene que haber estado presente por algunos meses, como lo revela el aumento del perínetro crameano (+2.2 D.S.). La sintomatología neurológica de sus últimos dias fue, sin dudas, originadid por la hemorragia cerebral y la compresión tumoral del quiasma óptico.

Polidipsia y poliuria, hiperosmolaridad plasmática y osmolaridad urinaria proporcionalmente baja son la traducción de una deficiencia parcial de ADH. ${ }^{*}$ Por razones obvias, el paciente no fue sometido a pruebas dinámicas en este sentido ni en la investigación de otras funciones homnonales.
El diagnóstico de certeza de Sindrome de Cushing se basó en las elevadas concentraciones de cortisol plasmático y su falta de supresión al suministrar $1 \mathrm{mg}$. de dexametasona la noche anterior. Esta dosis es mucho mayor que la recomendada de $20 \mu \mathrm{g} / \mathrm{Kg}$ de peso. ${ }^{\mathrm{y}}$ Paralelamente, la exsreción urinaria de 17 OHCS era exagerada, más de cuatro veces lo que se considera el límite máximo para su edad, peso y talla. ${ }^{10}$ Los 17 Ketosteroides estaban sólo discretamente aumentados.

El ritmo circadiano del cortisol plasmático puede ser demostrable a lus pocos meses de vida y está establecido casi en la totalidad de los niños al año de edad, "Iunque ocasionalmente puede manifestarse más tardianénte. ${ }^{12} \mathrm{Su}$ ausencia en nuestro paciente, por su edad, conforma un elemento de sospecha, pero no de certeza diagnóstica.

Los valores basales de Prolactina, LH, FSH y T4 estaban dentro de los límites normales. Es posible que estos valores representen la máxima capacidad hipotisiaria de secreción; solo el empleo de hormonas liberadoras u otros estímulos nos habrían podido demostrar la verdadera capacidad de reserva pituitaria.

Una determinación aislada de HGH carece de valor diagnóstico cuando la concentración encontrada es baja. En este caso podría pensarse en una eventual acción supresura del cortisol sobre los somatotrofos. Desde el punto de vista estatural, tan importante como ésta, es la acción que tienen los glucocorticoides sobre la generación de somatomedina y su acción sobre los cartilagos epifisiarios. ${ }^{10,13}$

El dosaje de ACTH plasmático, del que nu disponemos, es un elemento de gran utilidad en el diagnóstico del origen del $\mathbf{S}$. de Cushing. La concentración es baja en los casos de tumor suprarrenal y muy elevada en aquellos secundarios a producción ectópica de ACTH. De acuerdo a nuestra información, hay sólo una publicación de Enfermedad de Cushing en un niño menor con comprobación de concentración plasmática de $\mathrm{AC} \Gamma \mathrm{H}$ aumentadas y demostración de producción de ACIH in vitro por las células tumorales. ${ }^{3}$

La certificación de Enfenmedad de Cushing en nuestro paciente se basó en la comprobación necrópsica de un adenoma cromólobo de la hipófisis y una hiperplasia suprarrenal bilateral; el peso combinado de estas glándulas estaba aumentado en un $50 \%$ con respecto a lo normal.

Sólo una pequeña proporción de los casos de Enfermedad de Cushing tiene alteraciones radio- 
lógicas de la silla turca, y estos comesponden en su inayoria a adenomas cromótobos. ${ }^{2}$ Estos adenomas, a pesar de su aspecto a la microscopia de luz, pueden poseer gránulos secretorios demostrables a la microscopía electrónica o con métodos immunutluorescentes. ${ }^{3}$

El pronóstico del S. de Cushing en la infancia es polore. A pesar del tratamiento causal adecuado y de la sustitución esteroidal, la mortalidad es más del doble que la observada en adultos. ${ }^{14,15}$

\section{RESUMEN}

Se presenta el caso de un niño de 12 meses con Enfermedad de Cushing. Tenía los rasgos clásicos de hipercortisolismo, niveles elevados de cortisol plasınático que no suprimieron con dexametasona, excreción urinaria de 17 OHCS aumentada y una silla turca grande. Fallecíó poco después de su ingreso. Los hallazgos necrópsicos incluyeron una hemorragia intracraneana, un tumor hipofisiario con caracteres de ademoma cromófobo y una hiperplasia suprarrenal bilateral.

\section{REFERENCIAS}

1Wilkins, L. The diagunsis and treatunent of enducrine disorders in childhood and adolescence, second edition, Charles $C$. Thorvas, Sprinfield, 1965.
'Gixid, E.M. The Cushing Syndromes: changing views uf' didgnosis and tetutmeut. Ann. Intem. Med. 90: 829, 1979.

3Miller, W.L., Toumsend, J.J., Grambach, M.M. and Kaplan. S.L. An intant with Cushing's disease due to an adrenocurcotropun producing pituitary adenoma J. Clin. Endocrinol. Met:ab. 48: 1017, $197 \mathrm{y}$.

${ }^{4} M_{L}$ Arthter, $A$ C. Cloutier, M.D., Hagles, A.B. and Sprague, R.G. Cushung s disease in children mayo Clin. Proc. 47:318, 1972 .

SCilbert, M.G. and Cieteland, W.W. Cushing's syndrone in infancy Pedjatrics 46: 217 , 1970 .

SLoridan, L. and Senior, B. Cushing's syndrome in infancy J. Pediatr. 75: 349, 1969 .

${ }^{7}$ Cos, J., Matus, A, Dorantes, L., Salkado. M.M., de lus Monteros, A.E., Ajuria, M. y Chavarria, C. Sindrome de Cushingt en niños y adolescentes Bol. Med. Hosp. Infant. Mex. 31: 435. 3974.

BMilter, M., Dalakos, T, Moses, A.M., Fellerman, H, and Streeten, D.H.T. Recognition of partial defects in antidiuretic homone secretion Ann. Intern. Med. 73: 721, 1970.

${ }^{9}$ Tucci, J.R., Jagger, P.I., Lauler, D.P. and Thorn, G.W. Hapid dexamethasone suppresion test for Cusheng's syndronx JAMA 199: 379,1967 .

10Bondy, P.K. The adrenal cortex, in Metabolic control and disease, editado por Bondy P.K. Rosenberg. L.E., Philadelphia, W.B. Saunders, 1980 pp. $142 \bar{t}-1499$.

11 New, M.I. Comunicación personal.

I2f ranks, R.C. Diumal variation of plasma 17 Hydroxyeorticosteroids in children J. Clin. Endocrinol. Metab. 27: 75, 1967.

IJStreeten, D.H.P., Focs, F.H., Elders, M.J. Dalakos, T.G., Voorhess, M. Hipercotisolism in childhood: shoutconings of conventional diagnostic criteria Pediatrics 56: 797, 1975.

14 Orth, D.N. and Liddle, G.W. Hesults of treatment in $10 \mathrm{~s}$ p\&tients with Cushing's syndrome N. Engl. J. Med. 285: 243, 1971.

15Jennings, A.S., Liddit, G.W. and Orth, D.N, Hesults of treabing childhood Cushing"s disease with pituitary irradiation N. Eugl. J. Med. 297: 957 , 1977 . 